\title{
AVALIAÇÃO DO CONHECIMENTO DE PROFISSIONAIS DE ENFERMAGEM SOBRE A INJEÇÃO VENTROGLÚTEA
}

\author{
EVALUATION OF THE NURSES' KNOWLEDGE ABOUT \\ VENTROGLUTEAL INJECTION
}

\section{EVALUACIÓN DEL CONOCIMIENTO DE LAS ENFERMERAS RESPECTO DE LA INYECCIÓN VENTROGLÚTEA}

\author{
Fernanda Raphael Escobar Gimenes ${ }^{*}$ \\ Juliana Pereira Machado ** \\ Elisabeth Gulden Atila ${ }^{* * *}$
}

\begin{abstract}
RESUMO
Objetivo: Avaliar o conhecimento da equipe de enfermagem com relação à técnica de injeção intramuscular na região ventroglútea antes e depois de uma atividade de capacitação profissional. Método: Estudo quase-experimental, tipo antes e depois, entre janeiro e julho de 2010, em uma unidade de emergência em Descalvado-São Paulo, Brasil. A pesquisa foi realizada em três fases: Antes da intervenção, os participantes responderam um questionário específico para identificar seus conhecimentos teóricos sobre a técnica de injeção intramuscular na região ventroglútea. Na segunda fase, foi realizada uma atividade educativa com carga horária de 5 horas, com dois grupos de enfermeiros, com conteúdo teórico e prático. Na terceira fase, o conhecimento dos participantes foi reavaliado, após a capacitação. Resultados: Antes da intervenção, questões relacionadas às estruturas anatômicas envolvidas no mapeamento da região ventroglútea foram respondidas corretamente apenas $20,8 \%$ dos participantes, e após a intervenção, por 63,6\% deles. Além disso, após a intervenção houve 100\% de êxito na execução das 15 etapas contempladas na ferramenta de avaliação da prática. Conclusão: A intervenção de capacitação profissional foi efetiva em aumentar o conhecimento dos membros da equipe de enfermagem, mas ainda há falhas no uso de terminologias sobre estruturas musculares e ósseas envolvidas na delimitação da região ventroglútea.
\end{abstract}

Palavras chave: Avaliação, conhecimento, enfermagem, injeções intramusculares, capacitação profissional.

\begin{abstract}
Objective: To assess the knowledge of nursing staff regarding to the technique of intramuscular injection in the ventrogluteal site before and after professional training. Method: A single center, before-and-after study was carried out between January and July 2010 in an emergency unit in Descalvado-São Paulo, Brazil. The research was conducted in three phases. Before the intervention, participants were required to respond a questionnaire designed to identify their theoretical knowledge about intramuscular injection techniques in the ventrogluteal

*Enfermera. Professor Department of General and Specialized Nursing. University of São Paulo at Ribeirão Preto College of Nursing, Brazil. E-mail: fregimenes@eerp.usp.br

${ }^{* *}$ Enfermera. Professor. University of São Paulo at Ribeirão Preto College of Nursing, Brazil. E-mail: jpmachado311@gmail. com

${ }_{* * *}$ Enfermera. Newcastle Private Hospital, Newsouth Wales, Australia. E-mail: elisabethatila@yahoo.com.au
\end{abstract}


site. In the second phase, a five hour session of professional training was conducted to two groups of nurses and included theoretical and practical contents. In phase three, their knowledge was assessed, after having received the professional training. Results: Before the intervention, the question relating to the anatomical structures involved in mapping the ventrogluteal site were answered correctly by only $20.8 \%$ of the participants. However, after professional training, $63.6 \%$ of the participants correctly answered this question. Also, after the intervention, $100 \%$ successfully executed the 15 steps contemplated in the assessment tool. Conclusion: Professional training was effective in increasing the level of nurses' knowledge, but they still struggled with terminology regarding muscle and bone structures involved in mapping the site.

Key words: Evaluation, knowledge, nursing, injections, intramuscular, professional training.

\section{RESUMEN}

Objetivo: Evaluar el conocimiento del personal de enfermería en relación a la técnica de inyección intramuscular en la zona ventroglútea antes y después de la capacitación profesional. Material y método: Estudio de tipo antes y después realizado entre enero y julio de 2010 en una unidad de emergencia en Descalvado, São Paulo, Brasil. La investigación se realizó en tres fases. Antes de la capacitación se solicitó a los participantes que respondiesen a un cuestionario para identificar el conocimiento teórico acerca de la técnica de inyección intramuscular en la zona ventroglútea. En la segunda fase se llevó a cabo la capacitación profesional con una carga de trabajo de cinco horas a dos grupos de profesionales e incluyó contenidos teóricos y prácticos. En la tercera fase, el conocimiento de los profesionales fue reevaluado. Resultados: Antes de la intervención, la cuestión relacionada con las estructuras anatómicas implicadas en el mapeo de la zona ventroglútea fue respondida correctamente por solo el 20,8\% de los participantes. Después de la capacitación profesional, el 63,6\% de los participantes respondieron correctamente a esta pregunta. Además, el 100\% fue ejecutado con éxito las 15 etapas previstas en el instrumento de evaluación después de la intervención. Conclusiones: La capacitación profesional fue efectiva para aumentar el nivel de conocimiento de los profesionales de enfermería, pero tuvieron dificultades en la terminología relacionada con las estructuras musculares y óseas envueltas en el mapeo del sitio de la inyección.

Palabras clave: Evaluación, conocimiento, enfermería, inyecciones intramusculares, capacitación profesional.

Fecha recepción: 09/25/13. Fecha aceptación: 03/07/14.

\section{INTRODUÇÃO}

O tratamento medicamentoso destina-se a salvar vidas e melhorar a saúde. Portanto, todos os profissionais de saúde têm a responsabilidade de evitar danos desnecessários aos pacientes (1). As aplicações de medicamentos injetáveis são os procedimentos de cuidados de saúde mais comuns no mundo (2), e apesar de ser considerada uma técnica básica, está longe de ser inócuo. As consequências de falhas ou desconhecimento na prática durante a administração de medicamentos por injeção intramuscular (IM) podem colocar os pacientes em risco de incapacidade e morte (3).
Várias complicações estão associadas com injeções IM, especialmente aquelas administradas na região dorsoglútea. Estas incluem fibrose da pele e do tecido conjuntivo, fibrose e contratura muscular, paralisia de nervos, abcessos, gangrena, paralisia, e hemorragia em pacientes com distúrbios hemorrágicos (4-6).

Em nossa prática docente, durante a supervisão de estudantes de graduação em enfermagem, observamos o uso frequente da região dorsoglútea para injeções IM realizadas por equipe de enfermagem em unidade de emergência, mesmo quando o paciente já apresentou reações cutâneas ou musculares causadas pelo excesso de injeções nesse sí- 
tio. Nós, então, questionamos os fatores que contribuem para esta prática que é aparentemente repetitiva e executada sem a devida reflexão.

Embora a literatura de enfermagem dos últimos 20 anos recomende a região ventroglútea para injeções IM, é a dorsoglútea a região preferida pelos profissionais de enfermagem para a administração de medicação IM (7-9). A relutância em usar o site ventroglútea pode ser atribuída ao fato de que muitos profissionais de enfermagem não são treinados para esta técnica, contribuindo para a falta de confiança para realizar o procedimento (10). Isso mostra que a formação e educação dos profissionais de enfermagem ainda não mantém mesmo ritmo dos avanços na segurança do paciente, nem dos requisitos da força de trabalho (7).

\section{Referencial teórico}

De acordo com a Organização Mundial da saúde (OMS), cerca de 16 bilhões de injeções são administradas anualmente em todo o mundo, e assim, a importância de promover práticas seguras de injeção é vital (11). A injeção IM é caracterizada pela introdução da medicação dentro da fáscia muscular. Injeções IM são frequentemente administradas nas regiões: deltoide, vasto-lateral, e ventroglútea, dorsoglútea, onde medicamentos são rapidamente absorvidos. Por este motivo, a região IM é amplamente usada na prática clínica em serviços de saúde. Todos os músculos são inervados e tem um suprimento de sangue, mas apenas o local da injeção dorsoglútea está próximo a grandes nervos e vasos sanguíneos (8).

Embora injeções IM sejam procedimentos comumente realizados por profissionais de enfermagem, as técnicas utilizadas continuam enraizadas na prática $(8,10)$. Enfermeiros da prática clínica continuam a usar e instruir os estudantes de enfermagem a usar a região dorsoglútea como o local de escolha para injeções IM, apesar de fortes evidências sobre as complicações associadas a este local (9). As razões mais frequentemente declaradas para escolher esta região incluem acesso mais fácil, a maior extensão e volume desse músculo, a sensibilidade relativamente baixa para dor e o pedido do paciente. A falta de conhecimento dos profissionais de enfermagem sobre a delimitação anatômica da região ventroglútea e o acesso e às evidências publicadas sobre os benefícios de seu uso ( 9 , 12) representam um desafio para o avanço e promoção da utilização desta região para injeções IM.

A literatura apoia o uso da região ventroglútea em relação à dorsoglútea para administração de medicações via IM. No entanto, muitos enfermeiros não utilizam pesquisas conduzidas na área de enfermagem e nem relacionam com disciplinas teóricas como base para prática clínica na injeção IM. Sua fonte mais comum de conhecimento é um colega; muitas vezes se sentem mais confortáveis em pares para obter informações e conhecimento, do que buscando material científico baseado em evidências $(10,13,14)$.

As mesmas situações ocorrem no Brasil, onde as enfermeiras tendem a usar a região dorsoglútea para injeções IM. Estudo realizado em um hospital universitário no estado de São Paulo- Brasil mostrou que 65,6\% dos profissionais de enfermagem preferem usar a região dorsoglútea em vez da ventroglútea para injeções IM. De acordo com os investigadores, havia uma necessidade de atualizar o conhecimento do profissional sobre a terminologia usada para localizar a região, referências anatômicas, complicações associadas e as contraindicações das injeções IM (15).

Poucos estudos no Brasil avaliaram o conhecimento dos profissionais de enfermagem sobre injeções IM na região ventroglútea, no sentido de garantir resultados positivos para os pacientes e diminuir os riscos de eventos adversos relacionados. Além disso, até o momento não tivemos conhecimento de estudos que avaliaram o impacto de programas de capacitação profissional para aumentar a 
utilização da região ventroglútea para injeções IM.

A Organização Panamericana da saúde (OPAS) aponta para a necessidade de uma força-tarefa relacionada com a formação contínua dos enfermeiros, como meio de evitar os eventos adversos, reforçando assim a segurança do paciente. Neste contexto, há preocupação crescente relacionada com a educação dos profissionais de saúde, porque eles precisam entender como sistemas afetam a qualidade e segurança dos cuidados prestados (16).

A formação e capacitação de profissionais de saúde têm sido o alicerce para o cuidado seguro e de alta qualidade. No entanto, enfermeiros e outros profissionais de saúde não estão sendo adequadamente preparados para fornecer o melhor atendimento possível, e há também avaliação insuficiente de sua proficiência em curso. Programas de treinamento têm sido subutilizados e subvalorizados como uma ferramenta vital para enfrentar os desafios de melhorar a segurança do paciente. É claro que é necessária uma nova abordagem de educação e treinamento necessários para desempenhar plenamente seu papel e assim melhorar a segurança do paciente $(7,17)$.

O Instituto de medicina (17) cita também a importância de reformular os métodos de treinamento de saúde no sentido de enfatizar a qualidade e segurança dos cuidados. De acordo com o Instituto, há uma necessidade de explorar, definir e implementar modelos de formação profissional que não expõem os pacientes a riscos desnecessários. Há uma variedade de métodos educacionais que têm sido amplamente utilizados nos currículos dos cursos de graduação para melhorar a formação dos estudantes para a segurança do paciente, entre palestras, workshops, exercícios em ambiente de simulação, análise de causa raiz e outros métodos de aprendizagem interativa (18).

O uso de cenários de simulação, por exemplo, é um fenômeno global, e assim deve ser incentivado. Estes cenários contri- buem para a prática baseada em evidências e a tomada de decisão, permitem desenvolver conhecimentos, habilidades e atitudes a serem adquiridos de forma segura, eficiente e sem riscos para a segurança do paciente (19).

A formação profissional e atualizações dos conhecimentos permitem capacitar enfermeiros e profissionais de enfermagem para repensar e alterar seus as práticas atuais. Programas de educação continuada são fatores importantes para profissionais de enfermagem para adquirir conhecimentos e habilidades para administrar injeções IM no na região ventroglútea.

O objetivo deste estudo é avaliar o conhecimento e prática de profissionais de enfermagem, de uma unidade de emergência em um município brasileiro, sobre a técnica de injeção IM na região ventroglútea, antes e após um programa de capacitação profissional. Esperamos que a capacitação profissional possa minimizar a relutância de profissionais de enfermagem em usar a região ventroglútea, e que esses profissionais poderão adquirir mais confiança para realizar o procedimento, melhorando assim a prática clínica da enfermagem relacionada com as injeções IM.

\section{MÉTODO}

\section{Tipo de estudo}

Estudo quase experimental, do tipo antes e depois, em um único centro, com abordagem quantitativa.

\section{Amostra/participantes}

No Brasil, todas as categorias profissionais de enfermagem são habilitadas para administrar injeções IM. Então, todos os 45 profissionais de enfermagem de uma unidade de emergência, localizado em Descalvado-São Paulo, Brasil, foram convidados a participar de nos- 
so estudo. Vinte e quatro $(53,3 \%)$ aceitaram participar e assinaram um termo de consentimento. Destes, 6 (25\%) eram enfermeiros; $14(58,3 \%)$ eram técnicos de enfermagem e 4 $(16,7 \%)$ eram auxiliares de enfermagem. Foram incluídos profissionais com 18 anos de idade ou mais, e fossem autorizados a administrar injeções IM. No entanto, 13 (54,2\%) os participantes do estudo não concluíram o estudo devido a férias, demissão, e/ou afastamento durante a coleta de dados.

\section{Coleta de dados}

Os dados foram coletados entre janeiro de 2010 e julho de 2010. A pesquisa foi realizada em três fases.

\section{Instrumentos}

Para a coleta de dados foi utilizado um questionário estruturado autoaplicável, contendo oito questões, sendo cinco de múltipla escolha, e três abertas, adaptadas de um estudo anterior (17), relacionado ao conhecimento teórico sobre a técnica de injeção IM na região ventroglútea.

\section{Técnica de injeção IM}

Usamos a técnica descrita por Potter e Perry (20) para injeções IM na região ventroglútea como um guia para coleta de dados. Nela, o participante coloca a palma da sua mão sobre o grande trocânter do quadril cliente (mão direita sobre o quadril esquerdo, mão esquerda sobre o quadril direito). O polegar deve ser apontado para o cliente, e o dedo indicador sobre a espinha ilíaca anterossuperior e virilha. Em seguida, o participante deve estender o dedo médio ao longo da crista ilíaca em direção à nádega, para formar um triângulo entre o dedo indicador, dedo médio e crista ilíaca (em direção a nádega). A agulha deve ser inserida e o medicamento injetado no meio deste triângulo a um ângulo de 90 graus.

\section{Procedimentos para a coleta de dados}

Fase 1: Os participantes foram conduzidos a responder a um questionário elaborado para identificar seus conhecimentos teóricos sobre a técnicas de injeção IM na região ventroglútea. $\mathrm{O}$ instrumento foi respondido no próprio ambiente de trabalho, no momento mais conveniente. Em seguida, o conhecimento prático sobre técnicas de injeção IM foi avaliado através da observação direta não participativa. Os participantes teriam que demonstrar a técnica de acordo com o Potter e Perry (20) descrita anteriormente.

Foi utilizado um simulador estático, de baixa fidelidade, tipo manequim, e seringas e agulhas para simular a técnica, que ocorreu em uma sala privada, previamente organizada e replicada de forma idêntica, para avaliar a capacidade do participante em delimitar a região ventroglútea e observar a angulação e inserção da agulha.

Fase 2: Realizamos uma atividade educativa com carga horária total de 5 horas para dois grupos de profissionais de enfermagem, que incluía conteúdo teórico e prático idêntico e padronizado. As atividades aconteceram na sala de reuniões do hospital, em dias consecutivos para permitir a participação de profissionais de todos os turnos. O conteúdo teórico foi apoiado por recursos audiovisuais. Houve troca de experiências durante as discussões de ambos os grupos. Para a capacitação prática, foram usados: seringas, agulhas, algodão seco e embebido em álcool a 70\%, bandeja, luvas e um manequim simulador estático para praticar a técnica.

Nas três primeiras horas, fizemos explicações sobre injeções IM e nós dirigida apresentamos as razões para desenvolver a pesquisa. Também foram discutidos os aspectos éticos, legais e históricos relacionados com injeções IM, as vantagens e desvantagens de cada região, os critérios utilizados para escolher o a região adequada, e as características de cada estrutura muscular envolvida (deltoide, vasto 
lateral, dorsoglúteo, e ventroglúteo). O foco, no entanto, foi sobre a injeção IM na região ventroglútea. Nos duas horas subsequentes, abordamos os aspectos relacionados com o conteúdo prático: a identificação de estruturas ósseas envolvidas na região ventroglútea correta, a assepsia limpeza no local da injeção com um algodão embebido em álcool e a simulação da técnica no manequim simulador estático.

Fase 3: A coleta de dados pós intervenção foi realizada dentro de um período de 15 dias após o treinamento profissional (20). Portanto, seguimos a mesmas etapas e usamos os mesmos instrumentos da primeira fase.

\section{Validade e confiabilidade/rigor}

Nas fases 1 e 3, enquanto os participantes respondiam o questionário, permanecemos a uma proximidade física com o participantes para garantir que eles não consultaram alguma literatura, e para minimizar os riscos de esclarecimento de dúvidas ou troca de informações entre si, sobre o técnica de injeção IM na região ventroglútea.

\section{Análise estatística dos dados}

Para a comparação dos resultados antes e depois da intervenção educativa, foi utilizado o teste t pareado para explorar as diferenças estatísticas no conhecimento dos profissionais de enfermagem. O nível de significância foi fixado em $\mathrm{p}<0,05$.

\section{Aspectos éticos}

O estudo foi aprovado pelo comitê de ética da Universidade Camilo Castelo Branco (protocolo No 2654-2874/09). Todos os participantes receberam explicações sobre o estudo antes de participar e deram seu consentimento escrito antes de participação voluntária.

\section{RESULTADOS}

Nesse estudo foi avaliado o conhecimento dos profissionais de enfermagem, em uma unidade de emergência brasileira, antes e após uma intervenção educativa de capacitação teórica e prática sobre a técnica de injeção IM na região ventroglútea.

Por meio da utilização de questionário autoaplicável estruturado. Antes da atividade de capacitação profissional, quando questionados sobre as estruturas anatômicas envolvidas na localização e delimitação da região ventroglútea, a 20,8\% dos participantes respondeu com "crista ilíaca" somente, e $20,8 \%$ não responderam. No entanto, após a formação profissional, 63,6\% respondeu a pergunta corretamente: "crista ilíaca, espinha ilíaca anterior de Superior + grande trocânter" (Tabela 1).

A segunda questão refere-se aos músculos envolvidos na região ventroglútea. Apenas $4,2 \%$ dos participantes responderam à questão corretamente antes de formação profissional, em comparação com 36,4\% de respostas corretas após a formação profissional, com um aumento de 32,2\% (Tabela 2).

A Tabela 3 mostra a frequência de respostas corretas dadas pelos participantes durante a avaliação de seus conhecimentos teóricos, antes e depois da formação profissional. Houve um aumento no número de respostas corretas sobre a técnica de injeção IM na região ventroglútea, depois da formação profissional. Os resultados são considerados estatisticamente significativos, como o t-observado foi maior que o t-crítico $(6,67>$ $1,94)$, a $5 \%$ de probabilidade.

Quando os participantes foram convidados a descrever a técnica utilizada para localizar a região ventroglútea, antes da formação profissional, 29,2\% erraram a questão, e 70,8\% não souberam de responder. No entanto, observou-se que, mesmo depois de frequentar a formação profissional, nenhum 
Tabela 1. Respostas dos participantes $(n=11)$ sobre estruturas ósseas para localizar a região ventroglútea, antes e depois do programa de capacitação professional, Descalvado- Brasil, 2010 .

\begin{tabular}{lcccc}
\hline & \multicolumn{3}{c}{ Fase } \\
\cline { 2 - 5 } & \multicolumn{2}{c}{$\begin{array}{c}\text { Antes da } \\
\text { capacitação }\end{array}$} & $\begin{array}{c}\text { Depois da } \\
\text { capacitação }\end{array}$ \\
\cline { 2 - 5 } Estruturas ósseas & $\mathrm{n}$ & $\mathrm{n}$ & $\%$ \\
Crista ilíaca + Espinha ilíaca anterossuperior + Grande trocânter & 1 & 4,2 & 7 & 63,6 \\
Crista ilíaca + Espinha ilíaca póstero-superior & 1 & 4,2 & 0 & 0 \\
Crista ilíaca + Fossa ilíaca & 1 & 4,2 & 0 & 0 \\
Fêmur & 1 & 4,2 & 0 & 0 \\
Grande trocânter & 2 & 8,3 & 1 & 9,1 \\
Fossa ilíaca & 2 & 8,3 & 0 & 0 \\
Crista ilíaca + Grande trocânter & 3 & 12,5 & 1 & 9,1 \\
Espinha ilíaca anterossuperior & 3 & 12,5 & 0 & 0 \\
Crista ilíaca + Espinha ilíaca anterossuperior & 0 & 0 & 1 & 9,1 \\
Crista ilíaca + Espinha ilíaca antero-superior + Fêmur + Grande trocânter & 0 & 0 & 1 & 9,1 \\
Crista ilíaca & 5 & 20,8 & 0 & 0 \\
Não respondeu & 5 & 20,8 & 0 & 0 \\
\hline Total & 24 & 100 & 11 & 100 \\
\hline
\end{tabular}

Tabela 2. Respostas dos participantes $(n=11)$ sobre estruturas musculares encontradas na região ventroglútea, antes e depois do programa de capacitação professional, DescalvadoBrasil, 2010.

\begin{tabular}{lcccc}
\hline & \multicolumn{3}{c}{ Fase } \\
\cline { 2 - 5 } Estruturas musculares & \multicolumn{2}{c}{ Antes da capacitação } & \multicolumn{2}{c}{ Depois da capacitação } \\
\cline { 2 - 5 } Glúteo Médio + Glúteo Mínimo & $\mathrm{n}$ & $\%$ & $\mathrm{n}$ & $\%$ \\
Glúteo Mínimo & 1 & 4,2 & 4 & 36,4 \\
Glúteo Médio & 1 & 4,2 & 0 & 0 \\
Músculo tensor - fáscia lateral & 1 & 4,2 & 4 & 36,4 \\
Músculo Oblíquo Abdominal & 1 & 4,2 & 1 & 9,1 \\
Músculo Piriforme & 2 & 8,3 & 0 & 0 \\
Glúteo Máximo & 2 & 8,3 & 0 & 0 \\
Não respondeu & 4 & 16,7 & 1 & 9,1 \\
\hline Total & 12 & 50 & 1 & 9,1 \\
\hline
\end{tabular}


Tabela 3. Frequência de respostas corretas na avaliação do conhecimento teórico, antes e depois da capacitação profissional. Descalvado-Brazil, 2010.

\begin{tabular}{lcccc}
\hline & \multicolumn{4}{c}{ Fase } \\
\cline { 2 - 5 } & \multicolumn{2}{c}{$\begin{array}{c}\text { Antes da capacitação } \\
(\mathrm{N}=24)\end{array}$} & $\begin{array}{c}\text { Depois da capacitação } \\
(\mathrm{N}=11)\end{array}$ \\
\cline { 2 - 5 } Resposta correta* & $\mathrm{n}$ & $\%$ & $\mathrm{n}$ & $\%$ \\
Faixa etária preferencial para injeção ventroglútea & 15 & 62,5 & 10 & 90,9 \\
Ângulo da agulha & 8 & 33,3 & 11 & 100 \\
Possível decúbito para injeção ventroglútea & 6 & 25 & 10 & 90,9 \\
Desvantagens da região ventroglútea & 3 & 12,5 & 11 & 100 \\
Contraindicações da região ventroglútea & 2 & 8,3 & 11 & 100 \\
Músculos presentes na região ventroglútea & 1 & 4,2 & 4 & 36,4 \\
Estruturas ósseas relacionadas com a região ventroglútea & 1 & 4,2 & 7 & 63,6 \\
Descrição da técnica & 0 & 0 & 0 & 0 \\
\hline × Patcipantes poderayyyyyy
\end{tabular}

${ }^{*}$ Participantes poderiam escolher mais de uma resposta.

dos participantes foi capaz de descrever a técnica utilizada para delimitar a região ventroglútea corretamente. Os trechos abaixo ilustram como os profissionais a descrevem: "É definida de acordo com o ml deve ser administrado"; "Com o polegar localizado na crista ilíaca, que posteriormente ocupa o local do dedo médio, formando a dobra"; "Aplicam-se no meio da dobra".

Quando avaliamos respostas dos profissionais relativas aos músculos envolvidos na região ventroglútea, observamos $36,4 \%$ de respostas corretas após a formação profissional, contra $4,2 \%$ antes da intervenção. No entanto, observou-se que, mesmo após a atividade de capacitação profissional, nenhum dos participantes foi capaz de descrever a técnica utilizada para delimitar a região ventroglútea corretamente. Para a resposta a ser considerada correta, os participantes devem citar, pelo menos, as seguintes etapas: "Localize o ponto anterossuperior da espinha ilíaca com o dedo indicador e o ponto mais alto da crista ilíaca com o dedo médio; Localizar o trocanter e, depois de identificar o triângulo formado pelas estruturas, administrar a me- dicação no centro deste triângulo".

Para avaliar seus conhecimentos práticos, os participantes demonstraram a técnica através da simulação em um manequim simulador estático. Antes da atividade de capacitação profissional, dos 24 participantes, apenas $37,5 \%$ executaram a técnica, mas não foram capazes de fazê-lo corretamente. Os participantes que não executaram a simulação tinham pouco ou nenhum conhecimento sobre injeções IM na região ventroglútea. Após a capacitação, no entanto, todos os profissionais executaram com sucesso os 15 passos previstos no instrumento de avaliação (Tabela 4).

\section{DISCUSSÃO E CONCLUSÃO}

Um programa de capacitação profissional foi implementado em uma unidade de emergência brasileira com a finalidade de avaliar o conhecimento de profissionais de enfermagem sobre a injeção IM na região ventroglútea, antes e depois essa intervenção. Os resul- 
Tabela 4. Passos seguidos pelos participantes durante a simulação de injeção IM na região ventroglútea, antes e depois da capacitação profissional. Descalvado-Brazil, 2010.

\begin{tabular}{|c|c|c|c|c|c|c|c|c|c|}
\hline & \multirow[b]{3}{*}{ Passos da técnica } & \multicolumn{4}{|c|}{$\begin{array}{l}\text { Antes da capacitação } \\
\qquad(\mathrm{N}=24)\end{array}$} & \multicolumn{4}{|c|}{$\begin{array}{l}\text { Depois da capacitação } \\
(\mathrm{N}=11)\end{array}$} \\
\hline & & \multicolumn{2}{|c|}{ Correto } & \multicolumn{2}{|c|}{ Incorreto } & \multicolumn{2}{|c|}{ Correto } & \multicolumn{2}{|c|}{ Incorreto } \\
\hline & & $\mathrm{n}$ & $\%$ & $\mathrm{n}$ & $\%$ & $\mathrm{n}$ & $\%$ & $\mathrm{n}$ & $\%$ \\
\hline 1) & $\begin{array}{l}\text { Posicionou o simulador estático de acordo } \\
\text { com a posição supina que será usado }\end{array}$ & 8 & 33,3 & 1 & 4,2 & 11 & 100 & 0 & 0 \\
\hline 2) & $\begin{array}{l}\text { Colocou sua mão sobre o quadril (mão } \\
\text { direita sobre o quadril esquerdo, mão } \\
\text { esquerda sobre o quadril direito) }\end{array}$ & 0 & 0 & 9 & 37,5 & 11 & 100 & 0 & 0 \\
\hline 3) & $\begin{array}{l}\text { Localizou a espinha ilíaca anterossuperior } \\
\text { com o dedo indicador }\end{array}$ & 0 & 0 & 9 & 37,5 & 10 & 90,9 & 1 & 9,1 \\
\hline 4) & $\begin{array}{l}\text { Estendeu seu dedo médio ao longo da crista } \\
\text { ilíaca }\end{array}$ & 0 & 0 & 9 & 37,5 & 10 & 90,9 & 1 & 9,1 \\
\hline 5) & $\begin{array}{l}\text { Colocou a palma da mão sobre o trocânter } \\
\text { maior }\end{array}$ & 0 & 0 & 9 & 37,5 & 10 & 90,9 & 1 & 9,1 \\
\hline 6) & $\begin{array}{l}\text { Identificou o triângulo entre o dedo } \\
\text { indicador, dedo médio e crista ilíaca }\end{array}$ & 0 & 0 & 9 & 37,5 & 10 & 90,9 & 1 & 9,1 \\
\hline 7) & Fez a antissepsia do local & 4 & 16,7 & 5 & 20,8 & 9 & 81,8 & 2 & 18,2 \\
\hline 8) & Pegou a seringa & 7 & 29,2 & 2 & 8,3 & 9 & 81,8 & 2 & 18,2 \\
\hline 9) & Esticou a pele com uma mão & 0 & 0 & 0 & 0 & 0 & 0 & 11 & 100 \\
\hline 10) & $\begin{array}{l}\text { Introduziu a agulha ao longo do simulador } \\
\text { estático }\end{array}$ & 9 & 37,5 & 0 & 0 & 11 & 100 & 0 & 0 \\
\hline 11) & Introduziu a agulha em um ângulo de $90^{\circ}$ & 8 & 33,3 & 1 & 4,2 & 11 & 100 & 0 & 0 \\
\hline 12) & $\begin{array}{l}\text { Introduziu a agulha com um movimento } \\
\text { firme e único }\end{array}$ & 9 & 37,5 & 0 & 0 & 11 & 100 & 0 & 0 \\
\hline 13) & Simulou a administração de medicamento & 9 & 37,5 & 0 & 0 & 11 & 100 & 0 & 0 \\
\hline 14) & $\begin{array}{l}\text { Aplicou pressão usando uma bola de } \\
\text { algodão }\end{array}$ & 9 & 37,5 & 0 & 0 & 11 & 100 & 0 & 0 \\
\hline 15) & Retirou a agulha rapidamente & 9 & 37,5 & 0 & 0 & 11 & 100 & 0 & 0 \\
\hline
\end{tabular}

tados mostraram melhorias significativas nas respostas dos participantes após a capacitação. Estes resultados apoiam a proposta pela OPAS, que incentivou a introdução de programas de treinamento no local de trabalho, com o objetivo de melhorar a qualidade dos cuidados e segurança do paciente (16) .
Antes da intervenção, 20\% dos participantes não foi capaz de delimitar anatomicamente a região ventroglútea e a maioria deles respondeu incorretamente. Conclusões semelhantes foram detectadas em um estudo anterior (17), revelando pontos fracos no conhecimento de enfermeiros de uma área 
demográfica similar.

Mesmo após o programa de treinamento, os participantes não foram capazes de descrever a técnica corretamente, nem usar terminologia adequada para delimitar a região ventroglútea para injeções IM. Há uma clara necessidade de desenvolver estratégias para habilitar profissionais de enfermagem para melhorar seus conhecimentos e habilidades. Programas de capacitação visando à mudança de prática devem ser baseados em evidências e focados em desenvolver conhecimento, atitudes e limitações.

Um programa de educação por pares foi testado anteriormente (21) com o objetivo de desenvolver conhecimento e atitudes baseada em evidências em enfermagem pediátrica, em relação ao manejo da febre. De acordo com os pesquisadores, o programa facilitou a mudança de grupo e foi considerado adaptável a outras práticas, grupos e configurações. Nossos resultados também reforçam a tese de que a formação contínua e conhecimento atualizado poderão permitir ao profissional repensar e mudar sua prática. Instituições de saúde devem garantir que os pacientes recebam cuidados seguros e competentes (4) por meio de equipes bem treinadas e qualificadas.

Para garantir a prática segura na administração de terapia medicamentosa, enfermeiros devem usar julgamento clínico ao escolher o local da injeção, entendera relevância da anatomia e fisiologia, bem como os princípios para administrar uma injeção IM (6).

Se os enfermeiros não forem capacitados e treinados para usar técnicas de injeção IM ventroglútea adequadamente, faltará a confiança necessária para realizar o procedimento sem ajuda, porque é difícil colocar as informações que aprendeu em prática sem apoio e orientação (22).

É importante considerar que a região ventroglútea pode não ser tão segura quanto a dorsoglútea os enfermeiros tiverem dificuldade em localizar esta região anatomicamente (12). Também há necessidade de investi- mentos na formação, capacitação e educação permanente da enfermagem para a prática segura. Por esta razão, a Organização Mundial da saúde (7) vem explorando as ligações entre a educação dos trabalhadores de cuidados de saúde e a segurança do sistema de saúde. Também, educadores e organizações de certificação, licenciamento e acreditação hospitalar, devem assegurar que os estudantes e profissionais desenvolvam e mantenham a proficiência (17).

Apesar de nenhum participante ter sido capaz de descrever corretamente a técnica usada para delimitar a região ventroglútea, todos foram capazes de simular a técnica corretamente, após a intervenção de capacitação profissional. Há um conflito na enfermagem em relação à prática e o respectivo conhecimento teórico. Profissionais precisam ser capacitados para saberem avaliar e valorizar todas as fontes de conhecimento e evidências, e é vital que se tornem pensadores críticos, e com isso, possam aumentar a quantidade e a qualidade dos cuidados prestados (23).

Para melhorar a capacitação e, consequentemente melhorar a segurança do paciente, os profissionais de saúde estão utilizando treinamentos de simulação em muitas formas. Treinamento com simulação pode ser usado em hospitais e outras instituições de saúde com uma série de razões, incluindo o treinamento de trabalho e equipe, avaliação de competências e como treinamento de habilidades e competências para iniciantes $(16,24)$. É uma ferramenta poderosa de aprendizagem para ajudar o profissional da saúde moderno a atingir níveis mais elevados de competência e assistência mais segura, e também elimina os riscos inerentes a ao treinamento de cuidados em pacientes vivos (19).

Outros estudos mostram que, treinamentos bem delineados baseados em simulação, podem fornecer a estrutura para o aprendizado de intervenções críticas e complexas, tais como a administração de medicamentos $(25,26)$. Sessões de treinamento intensivo 
deste tipo podem melhorar a capacidade e a habilidade de enfermeiros para a administração injeções IM na região ventroglútea.

Nosso estudo teve algumas limitações. Existem diferenças na experiência e formação dos profissionais de enfermagem de uma região para outra, e o pequeno número de participantes na amostra não permitiu a generalização dos resultados obtidos neste estudo. Seriam necessários maiores tamanhos de amostra. Não houve, até o momento, estudo controlado randomizado em amostras semelhantes para comparar os resultados do estudo.

Também, o tipo de estudo, "antes e depois", que é amplamente utilizado em investigações clínicas, proporciona aos participantes a uma situação onde eles são conscientemente observados. Um fenômeno conhecido como efeito Hawthorne pode resultar em uma mudança positiva e intencional no comportamento dos participantes, uma vez que sabem que estão sendo observados (27). Além disso, os participantes podem ter atitudes divergentes daquelas realizadas na prática clínica.

Este estudo permite concluir que a formação profissional foi eficaz em aumentar o nível de conhecimento dos profissionais de enfermagem sobre a técnica de injeção IM na região ventroglútea, porém ainda permaneceram dificuldades no uso de terminologias corretas sobre estruturas musculares e ósseas contidas na descrição da técnica.

Em conclusão, acreditamos que promover programas de educação continuada configura-se em aspecto importante para profissionais de enfermagem para adquirir habilidades para administrar medicamentos através de injeções IM na região ventroglútea, de forma segura. $\mathrm{O}$ uso da região dorsoglútea para injeções IM deve ser removido da prática, pois representa um risco desnecessário e inaceitável para os pacientes.

Há necessidade de mais pesquisas com foco em métodos de capacitação baseados em evidências, que foquem tanto a teoria quanto a prática, para melhorar o conheci- mento e habilidades para administrar injeções de IM seguras.

\section{REFERÊNCIAS}

1. World Health Organization. WHO best practices for injections and related procedures toolkit. Geneva: World Health Organization; 2010.

2. Ağaç E, Güneş UY. Effect on pain of changing the needle prior to administering medicine intramuscularly: a randomized controlled trial. J Adv Nurs. 2011; 67(3): 563-568.

3. Plotkin SA, Orenstein WA, Offit PA, editores. Vaccines. 5a ed. Philadelphia: Saunders; 2008.

4. Walsh L, Brophy K. Staff nurses' sites of choice for administering intramuscular injections to adult patients in the acute care setting. J Adv Nurs. 2011; 67(5): 1034-1040.

5. Cocoman A, Murray J. Intramuscular injections: a review of best practice for mental health nurses. J Psychiatr Ment Health Nurs. 2008; 15(5): 424-434.

6. Hunter J. Intramuscular injection techniques. Nurs Stand. 2008; 22(24): 35-40.

7. World Health Organization. WHO Patient Safety. Patient safety curriculum guide: multi-professional edition. Geneva: World Health Organization; 2011.

8. Malkin B. Are techniques used for intramuscular injection based on research evidence? Nurs Times. 2008; 104(50-51): 48-51.

9. Cocoman A, Murray J. Recognizing the evidence and changing practice on injection sites. Br J Nurs. 2010; 19(18): 11701174.

10. Carter-Templeton H, McCoy T. Are we on the same page?: a comparison of intramuscular injection explanations in nursing fundamental texts. Medsurg Nurs. 2008; 17(4): 237-240. 
11. World Health Organization. Injection safety. Geneva: World Health Organization; 2006.

12. Wynaden D, Landsborough I, McGowan S, Baigmohamad Z, Finn M, Pennebaker D. Best practice guidelines for the administration of intramuscular injections in the mental health setting. Int J Ment Health Nurs. 2006; 15(3): 195-200.

13. Nicoll LH, Hesby A. Intramuscular injection: an integrative research review and guideline for evidence-based practice. Appl Nurs Res. 2002; 15(3): 149-162.

14. Pravikoff DS, Tanner AB, Pierce ST. Readiness of U.S. nurses for evidence-based practice. Am J Nurs. 2005; 105(9): 40-51; quiz 2.

15. Godoy S, Nogueira MS, Mendes IA. Intramuscular drug administration: analysis of knowledge among nursing professionals. Rev Esc Enferm. USP. 2004; 38(2): 135-142.

16. Cometto MC, Gómez PF, Sasso GTMD, Grajales RAZ, Cassiani SHDB, Morales $\mathrm{CF}$, editores. Enfermería y seguridad de los pacientes. Washington, DC: Organización Panamericana de la Salud; 2011.

17. Peterson C. Health professions education: a bridge to quality. Tar Heel Nurse. 2003; 65(4): 12.

18. Kiersma ME, Plake KS, Darbishire PL. Patient safety instruction in US health professions education. Am J Pharm Educ. 2011; 75(8): 162.

19. Aggarwal R, Mytton OT, Derbrew M,
Hananel D, Heydenburg M, Issenberg B, et al. Training and simulation for patient safety. Qual Saf Health Care. 2010; 19(Suppl 2): i34-43.

20. Potter P, Perry AG. Fundamentos de enfermagem. 8 ed. Rio de Janeiro: Elsevier; 2013.

21. Edwards MT. The objective impact of clinical peer review on hospital quality and safety. Am J Med Qua. 2011; 26(2): 110119.

22. Greenway K. Using the ventrologluteal site for intramuscular injection. Nurs Stand. 2004; 18(25): 39-42.

23. Hall A. Defining nursing knowledge. Nurs Times. 2005; 111(48): 1.

24. Nishisaki A, Keren R, Nadkarni V. Does simulation improve patient safety? Self-efficacy, competence, operational performance, and patient safety. Anesthesiol Clin. 2007; 25(2): 225-236.

25. Pauly-O'Neill S. Beyond the five rights: improving patient safety in pediatric medication administration through simulation. Clinical Simulation in Nursing. 2009; 5(5): e181-e186.

26. Ford DG, Seybert AL, Smithburger PL, Kobulinsky LR, Samosky JT, Kane-Gill SL. Impact of simulation-based learning on medication error rates in critically ill patients. Intensive Care Med. 2010; 36(9): 1526-1531.

27. Fletcher RH, Fletcher SW. Clinical epidemiology: The essentials. Baltimore: Lippincott Williams \& Wilkins; 2013. 